\title{
HIGHLIGHTS
}

HEMATOLOGY

\section{Cancer-initiating cells identified in acute promyelocytic leukemia}

Recent evidence has identified the murine equivalent of the elusive cancer-initiating 'stem' cells thought to be responsible for relapse in patients with acute promyelocytic leukemia (APL). These cells, described in Blood by Florence Guibal and colleagues, can initiate and propagate disease by persisting in hematopoietic organs of mice with APL. "The cancer 'stem' cell hypothesis proposes that relapses in [APL] and other cancers might be the result of inefficient therapeutic targeting of a small fraction of cells responsible for initiating and/or maintaining the bulk population of cancer cells," say the authors.

The investigators used an APL mouse model in which cells isolated from the spleens of leukemic transgenic mice were transplanted into isogenic recipients, an effect that mimics human APL disease. The researchers fractionated cells_- using a novel cell-sorting approach-from the bone marrow and spleen of both APL and wild-type mice into distinct stages of myeloid differentiation and found considerably more c-kit ${ }^{+} / \mathrm{CD} 34^{+}$cells in the APL mice. These cells (that also had a FcR $\gamma \mathrm{III} / \mathrm{II}^{+}, \mathrm{Gr} 1^{\text {int }}$ phenotype) were capable of generating leukemia when injected into sublethally irradiated control mice and the researchers noted a 100-fold increase in the number of leukemiainitiating cells in this cell population when compared with unfractionated leukemic bone marrow cells.

\section{4 ...the researchers noted a} 100 -fold increase in the number of leukemia-initiating cells in this cell population... 77

Furthermore, the investigators observed decreased expression of CCAAT/ enhancer binding protein $\alpha(\mathrm{C} / \mathrm{EBP} \alpha)$ in the leukemia-initiating cells when compared with control cells. As $\mathrm{C} / \mathrm{EBPa}$ is an essential transcription factor for myeloid differentiation, the authors suggest that down-regulation of $\mathrm{C} / \mathrm{EBPa}$ might be involved in the pathogenesis of
APL in this model. Indeed, they identified a role for promyelocytic-retinoic acid receptor a (a fusion protein associated with APL in humans) in C/EBPa downregulation in human APL cell lines. In addition, increased APL development and subsequently higher mortality was observed in $\mathrm{C} / \mathrm{EBPa}^{+/-}$mice expressing human promyelocytic-retinoic acid receptor $a$ when compared with control mice.

"Identification of APL cancer-initiating cells will lead to a greater understanding of the mechanisms underlying the initiation of APL" conclude the investigators, who go on to question whether "such understanding will lead to [the] design of novel treatments targeting the residual cancer-initiating cells postulated to be responsible for relapse after effective treatment."

\section{Rowan Higgs}

Original article Guibal, F. C. et al. Identification of a myeloid committed progenitor as the cancer-initiating cell in acute promyelocytic leukemia. Blood 114, 5415-5425 (2009) 